\title{
Le mauvais ton de Stendhal
}

Les comptes rendus du Rouge et le Noir en 1830-1831

\section{Pierre Laforgue}

\section{(2) OpenEdition}

\section{Journals}

Édition électronique

URL : http://journals.openedition.org/recherchestravaux/362

DOI : $10.4000 /$ recherchestravaux.362

ISSN : 1969-6434

Éditeur

UGA Éditions/Université Grenoble Alpes

Édition imprimée

Date de publication : 15 juillet 2009

Pagination : 171-179

ISBN : 978-2-84310-146-5

ISSN : 0151-1874

Référence électronique

Pierre Laforgue, «Le mauvais ton de Stendhal », Recherches \& Travaux [En ligne], 74 | 2009, mis en ligne le 28 février 2011, consulté le 08 septembre 2020. URL : http://journals.openedition.org/ recherchestravaux/362 ; DOI : https://doi.org/10.4000/recherchestravaux.362 
Pierre LAForgue

Université de Franche-Comté

\section{Le mauvais ton de Stendhal Les comptes rendus du Rouge et le Noir en 1830-183I}

L'affaire est entendue : Stendhal écrit mal, et il écrit mal parce qu'il n'a pas de style ou parce qu'il ne soigne pas son style. Il n'a pas de style : lui-même a avoué lire une page du Code civil chaque jour, avant de commencer à écrire, pour obtenir le degré de sécheresse convenable et se prémunir contre les fausses élégances du beau style romantique; il ne soigne pas son style : Balzac dans son grand article sur La Chartreuse le lui reproche vertement et donne des exemples de ses négligences, etc. Ce genre de considérations ne mène pas à grand-chose, sinon à la critique du bon goût, c'est-à-dire au j'aime/j'aime pas, et aux jugements de valeur dont ont été victimes un certain nombre d'écrivains. Dans le meilleur des cas, cela ne peut présenter un intérêt que dans le cadre d'une étude du bien écrire à une époque donnée.

Ce n'est pas la perspective que nous adopterons. Notre propos sera d'examiner comment les contemporains de Stendhal ont recouru à cette catégorie, chez eux assez élastique, du style, pour rendre compte de ses livres, en particulier de ses romans; comment, à travers cette question du style stendhalien, se problématisent des interrogations de tous ordres, politique, social, idéologique, littéraire. Pour cela nous nous attacherons aux comptes rendus du Rouge et le Noir entre novembre I 830 et février I 83 I $^{1}$, parce qu'ils sont nombreux et offrent une matière abondante, très diversifiée, parce que ce roman a eu un succès très important, et qu'il a définitivement donné son identité littéraire à Stendhal comme «l'auteur de Ronge et Noir» ou «l'auteur du Ronge et Noir». Il ne s'agit pas de faire une étude de réception du roman², même si,

I. Ces comptes rendus ont été réunis et publiés par V. Del Litto dans son ouvrage, Stendhal sous l'ail de la presse contemporaine (I8I7-I843), Champion, «Textes de littérature moderne et contemporaine», 200I. Toutes nos références y renverront.

2. Pour une étude de réception du Ronge, voir Stendhal, préface de M. Crouzet, Presses de l'université de Paris-Sorbonne, «Mémoire de la critique», I996, p. I3-17. 
inévitablement, nous serons amené à traiter de sa réception, mais de montrer comment, dans la plupart des comptes rendus, surgissent des jugements sur le style de Stendhal, et de voir ce qui est impliqué dans ces jugements, ceux-ci étant eux-mêmes fort peu stylistiques et ne se préoccupant absolument pas des stylèmes stendhaliens. Ce qui nous guidera, bien au contraire, c'est la mise en demeure du critique de la Gazette de France, terminant son compte rendu du roman par ces mots : «il est plus que temps que M. de Stendhal change $[. .$.$] pour toujours de manière et de style» (p. 613). Pourquoi? parce$ que M. de Stendhal a mauvais ton et que dans ce mauvais ton, fait d'ironie, d'insolence et d'impertinence, se révèle une attitude idéologiquement et politiquement incorrecte.

Notre point de départ sera le quasi-postulat sur lequel reposent à peu près tous les comptes rendus du Ronge et le Noir, à savoir l'assimilation du style à l'auteur. En l'occurrence, «le style, c'est l'homme». Ce jugement de Buffon, les critiques de i 830 le prennent implicitement à leur compte : presque aucun article consacré au Rouge et le Noir ne s'épargne des considérations sur l'auteur. C'est que faire un portrait moral de celui-ci, c'est expliquer son roman et le style de son roman. Son portrait moral est facile à faire, il tient en un seul mot, celui d'esprit. Ce substantif et l'adjectif de spirituel se rencontrent constamment dans tous les comptes rendus du Ronge. Stendhal est le «spirituel auteur des Promenades dans Rome» (p. 567), c'est un «homme d'esprit» (p. 572), etc. Dans un des comptes rendus les plus intelligents, celui d'Anselme Pétetin pour la Revue encyclopédique, on peut lire ce jugement, très révélateur de l'identification de l'auteur à son style à laquelle procède la critique :

L'homme spirituel qui se cache sous ce nom a déjà fait ses preuves ailleurs : il serait difficile d'être plus brillant, plus piquant, plus original. Mais, dans ce genre léger, il n'a peut-être été donné qu'au seul Voltaire de ne jamais tomber dans la manière et l'affectation : c'est un malheur que n'a pu éviter M. de Stendhal. (p. 62I)

Nous finirons sur l'assimilation de l'auteur à son style, en mentionnant un exemple-limite, celui qui est donné par Janin dans sa longue recension pour le Journal des Débats. L'identification de l'auteur au style est ici telle que le courriériste s'en prend à la personne même de Stendhal, et une page entière est occupée par une diatribe passionnée, presque haineuse, à faire un portrait répulsif de lui. L'enjeu n'en est pas psychologique, mais critique. Nous en extrayons le passage suivant :

C'est un observateur à froid, un railleur cruel, un sceptique méchant, qui est heureux de ne croire à rien, parce qu'en ne croyant pas, il a le droit de ne rien respecter et de flétrir tout ce qu'il touche. (p. 599) 
Observation, raillerie, scepticisme : ce sont autant de caractéristiques du style de Stendhal.

Un auteur spirituel ne peut donc que produire un ouvrage spirituel, qui illustre son «bon goût» (p. 621), qui montre des «aperçus fins» (p. 573), des pensées ingénieuses, un coloris original, des pages chaleureuses (p. 572). Ces appréciations se résument chez le critique de L'Artiste en deux phrases très élogieuses :

[...] il y a de l'esprit, de l'esprit fin, des choses vues et décrites à la loupe, une immense combinaison de petits riens qui forment je ne sais quoi qui plaît. C'est de la jaserie exquise, une manière de profondeur qui porte sur des détails sans consistance. (p. 6r4)

la pratique de l'esprit devant être considérée comme l'expression d'un je ne sais quoi dix-huitiémiste, qui rappelle l'art de la conversation dans les salons de l'Ancien Régime. En quoi Le Ronge et le Noir est, selon le jugement d'Anselme Pétetin, «un livre d'aristocratie» (p. 622), qui se signale par un "persiflage admirable de sel et d'esprit». Cette idée d'une filiation dix-huitiémiste de Stendhal se retrouve assez souvent. Le critique du Figaro, tout en s'attardant sur le personnage proprement extraordinaire de Mathilde, signale cependant, comme en passant, «ces enluminures, ces silhouettes dorées, admirablement placées dans une table de chapitres sous le titre de : la Vieille épée, le Vase du Japon, le Tigre, etc.» (p. 587). En cela il est un bon stendhalien, sensible à ce mélange caractéristique chez notre auteur de romantisme passionné et d'élégance d'Ancien Régime.

Rien d'étonnant, dès lors que l'on voit dans un Stendhal un écrivain spirituel et qui manie l'esprit, que son roman soit qualifié de "piquant» (p. 570), ou, bien plus souvent, d'«original». Ce second qualificatif est récurrent: on ne cesse de relever «l'originalité connue du style de l'écrivain» (p. 572). Cette originalité est constamment relevée, en bonne ou en mauvaise part. Généralement, c'est un critère relativement négatif. Ainsi sont associés originalité et goût du paradoxe : Stendhal est un «ami des paradoxes» (p. 571), un «faiseur de paradoxes» (p. 599). Le paradoxe est une saillie de l'esprit, mais il faut s'en méfier: il peut quelquefois être imputé au seul «caprice de l'auteur» (p. 580 ), et faire naitre des bizarreries. On stigmatise alors chez lui les «inventions les plus bizarres de son ouvrage» (p. 585 ), et on lui reproche de ne viser qu'à l'imprévu. L'imprévu résulte directement de l'originalité, et suscite de la part des critiques des mises en garde :

Puissent certains auteurs se guérir de la manie la plus dangereuse, celle de viser à l'originalité, de faire des mours et des passions avec de l'esprit et de viser en tout à l'imprévu». (p. 584) 
Ce jugement est précieux, il réunit l'originalité, l'esprit et l'imprévu et permet de comprendre comment se problématisent ces trois notions les unes par rapport aux autres. L'esprit fait naitre l'originalité et celle-ci suscite l'imprévu. L'imprévu chez Stendhal est un fait de style, le critique de L'Artiste organise même l'ensemble de son compte rendu autour de cette idée. D'entrée de jeu, il déclare : «L'art de M. de Stendhal, c'est le désappointement» (p. 613). Désappointement n'est pas ici pris en trop mauvaise part, c'est presque l'équivalent de la déception barthésienne; il poursuit : «son but, son tic, son mot favori, l'imprévu» (p. 613), pour conclure, mi-figue mi-raisin, à propos de Mathilde et de ses fantasmagories du XVI ${ }^{\mathrm{e}}$ siècle : «Je ne sais rien de moins naturel; mais c'est bizarre» (p. 6I4). De nouveau, un couplage passionnant de mots : naturel versus bizarre. Le bizarre, comprend-on, est la conséquence de la poétique de l'imprévu que Stendhal pratique dans son roman; cette poétique de l'imprévu provient de l'originalité intellectuelle de l'auteur. La contrepartie, c'est l'absence de naturel. C'est une approche qui est complètement antistendhalienne, mais elle a sa logique, du moment que l'on mobilise le concept d'originalité. Le critique de L'Artiste n'est pas un cas isolé; d'autres de ses confrères suivent la même voie. Celui de la Gazette littéraire commence par ces mots : «Voici un titre distingué. Le livre l'est aussi» (p. 580). Ce n'est pas du tout un compliment, au vu de la tirade polémique qui vient à la suite, dirigée, avec plus de bêtise que d'intelligence, contre le snobisme des bobos de I 830 et qui aboutit à cette conclusion :

Heureusement que M. de Stendhal a senti ce que devait être un livre au XIx ${ }^{e}$ siècle. C'est dans ce but qu'il a travaillé, faisant usage à la fois de son talent et de l'entente qu'il a des goûts du siècle. Aussi je ne connais pas de livre plus inégal en fait de style, de caractères, d'intrigues, de discussions et d'intérêt.

Ce rejet de Stendhal est celui du XIX ${ }^{\mathrm{e}}$ siècle lui-même et l'animosité qui motive cette mauvaise humeur, dont l'auteur du Ronge et le Noir fait les frais, ne nous importe pas; seul compte que les raisons, parfaitement idéologiques, de ce rejet suscitent un jugement sur le style. Un tel jugement sur le style a des implications elles-mêmes littéraires; ainsi dans cet article, le recenseur, au mépris du texte de Stendhal, se laisse aller à mentionner de prétendus «détails qui seront sublimes pour les amateurs de scènes de la place de Grève» (p. 584), alors que justement le roman est d'une totale discrétion sur ces détails. Pour notre critique, c'est un moyen facile et malhonnête de revenir une nouvelle fois sur l'idée des méfaits de l'originalité, ce qui le pousse à écrire : «L'auteur aurait pu à toute force en rester là; mais le dénouement eût été commun» (p. 584), et à suggérer une fin pleine d'hémoglobine. Hémoglobine romantique, cela va de soi, ce qui suffit à disqualifier un pareil roman. Il n'y a que Mme de Rênal qui échappe aux accès de malveillance ironiques du critique : 
«C'est là qu'est le naturel le plus parfait; et il est développé avec un talent supérieur» (p. 585). Plus généralement, l'absence de naturel est un des chevaux de bataille des critiques du Ronge en i 830, le critique de la Revue de Paris, comme celui de la Gazette littéraire, mais plus intelligemment, en fait grief à Stendhal (p. 6oI) et il lui reproche ce qu'il appelle «le cherché» (p. 6o2), quand il n'emploie pas l'expression, tout aussi maladroite et malheureuse, «le plus grand que nature» (p. 610).

En résumé, l'esprit mène à l'originalité et celle-ci à l'imprévu, au distingué, au cherché, etc.; mais l'esprit, c'est aussi l'intellectualisme, contre le sentiment, contre l'épanchement romanesque et l'effusion sentimentale C'est là un des leitmotive des comptes rendus du Rouge : le talent, ou la manie, de l'analyse chirurgicale par Stendhal : il est un «opérateur» (p. 592), travaillant dans un «amphithéâtre» (p. 592, 60I), occupé à « disséquer» (p. 604) ses personnages, avec un «scalpel» (p. 6I 2), ou les examinant avec une «loupe» (p. 592). Ce n'est pas un écrivain, mais un anatomo-pathologiste. Apostrophant ce singulier médecin légiste, Janin, dans le Journal des débats, lui déclare : «plus tard et à ton préjudice viendront le poète ou le romancier qui feront leur devoir» (p. 592). Clairement, Stendhal va à l'encontre du code littéraire et n'écrit pas un roman. Cela tient à ce qu'il «y parle passion avec l'esprit plutôt qu'avec le cœur», remarque le critique du Globe. Le propre de son style, c'est «la sécheresse de la pensée» (p. 6I4), autrement dit la rigueur, la précision de l'analyse. Aussi lorsqu'un recenseur veut être aimable, ne recourt-il pas à la métaphore médicale, mais voit en lui «le Larochefoucault [sic] des romanciers, [...] presque le Machiavel» (p. 604). C'est donc dans ce cas-là un moraliste, un romancier déviant, hors normes. Et justement ce qui caractérise ce romancier, c'est son style, qui n'est pas celui d'un romancier, du moins un romancier pour femmes de chambre. Le roman lui-même se ramène au style. Passant à l'examen de la seconde partie du Rouge, un critique a cette formule significative : «Alors commence un autre livre, un autre style» (p. 583 ).

La Rochefoucauld, Machiavel, - et aussi Voltaire. Même si son nom n'est pas très souvent mentionné de manière explicite dans les comptes rendus du Ronge, il n'y est guère question que de lui. Sur lui se fixent toutes sortes de références, stylistiques aussi bien qu'idéologiques, qui renvoient, pêle-mêle, à l'esprit, à l'incroyance, au culte de la raison, au XVIII' siècle pré-révolutionnaire, etc. En fait, chaque fois qu'il est question de l'esprit de Stendhal, c'est de Voltaire qu'il s'agit, «Voltaire» avec des guillemets, comme représentant des Lumières, "d'exécrable mémoire», dirait De Cholin dans Le Rouge, comme écrivain qui par son style piquant et ironique, irrespectueux de ce qu'il faut respecter, a sapé l'ordre des choses. Stendhal est l'un de ses héritiers... en i 830. 
Ce à quoi ont été sensibles les critiques du Ronge, et ce qui les a déconcertés ou irrités ou ce qui, quelques rares fois, leur a plu, c'est la rencontre de deux régimes d'historicité, l'un qui vient d'un $\mathrm{XVIII}^{\mathrm{e}}$ siècle antérieur à la Révolution, l'autre, immédiatement contemporain, celui de i 830 . C'est dans la confrontation critique de ces deux régimes d'historicité qu'est à chercher, aux yeux des contemporains de Stendhal, la singularité du Ronge et le Noir, poétique et romanesque, philosophique et politique. C'est une tâche ardue, et d'autant plus que Stendhal peut difficilement passer pour l'un de ces écrivains romantiques, comme Hugo, que leurs prises de position militantes permettent d'identifier immédiatement dans le champ de la critique et qui se prêtent sans peine aux développements les plus convenus et les plus prévisibles. De là, en ce qui concerne Stendhal, les contorsions intellectuelles auxquelles sont amenés les critiques. L'un des effets positifs de cela est que, pour les plus sagaces d'entre eux, une véritable réflexion est engagée sur les enjeux politiques et historiques d'une littérature qui produit des romans aussi inclassables que Le Rouge et le Noir. Cela nous conduira en conclusion à examiner comment, à propos du style de Stendhal, ont pu être posées des questions d'ordre littéraire, où était engagée une réflexion d'ordre idéologique. (Précisons au passage qu'il n'y a pour ainsi dire pas d'attaques contre le libéralisme de Stendhal, sauf chez quelques légitimistes grincheux, qui savent qu'ils ont affaire à un libéral. Comme quoi ce n'est pas sur le plan politique que se placent les choses.)

Comment donc les événements de Juillet ont-ils modifié la pratique de la littérature et qu'est-ce que cette modification signifie véritablement? Pour répondre à cette question, nous nous appuierons sur l'article le plus intelligent, et de loin, celui du Globe, où cette question est posée en ces termes. Nous citerons largement, tant la réflexion est intéressante :

C'est dans les époques de révolution, où la société s'agite en proie aux convulsions et aux angoisses, où la vie des individus comme celle des nations n'est que malaise, tourments et douleurs, qu'apparait cette poésie qui s'acharne à étaler dans leur hideuse nudité les plaies dont l'homme est affligé, de cette poésie qui commence à Shakspeare [sic], qui, par les Confessions de Rousseau et les sarcasmes de Diderot, est venue jusqu'à nous, dicter à M. de Chateaubriand les gémissements de René et s'exhaler dans les soupirs de la muse romantique.

C'est que chacun, isolé et marchant sur des ruines, ne trouve plus dans ses yeux que des larmes, et dans son cœur que de l'amertume; c'est que la société n'a plus qu'une vie galvanique, c'est qu'en elle tout mouvement n'est plus que la convulsion d'une agonie, c'est que le siècle où nous vivons aura cessé avant que l'horloge séculaire ait sonné sa dix-neuvième heure. (p. 573-574)

S'agissant du Ronge et le Noir, "Chronique du XIX ${ }^{e}$ siècle», c'est une analyse très juste. Le critique du Globe met parfaitement en évidence l'influence directe 
des conditions historiques sur la pratique de l'écriture romantique, et sur l'apparition elle-même du romantisme. Celui-ci n'est pas une modification de la sensibilité littéraire, mais la réaction au changement historique ayant affecté la société; plus loin dans le compte rendu sera explicité le divorce qui s'est produit entre la société et les «hommes au cœur plus grand que leur fortune» (p. 575), tel Julien, mais ce qui est ici essentiel est que le romantisme est pensé non pas dans une perspective littéraire, mais historico-politique. Les références littéraires qui sont données, de Shakespeare à Chateaubriand, ont valeur de symptôme, celui du malaise, du mal-être social, qui se traduit par une mutation de la sensibilité, et que l'on appelle aussi mal du siècle. Très intelligemment, le critique du Globe inscrit Stendhal dans cette mutation de la sensibilité, mais n'en fait pas pour autant un épigone de Chateaubriand, ce qui serait absurde; ce que Chateaubriand et Stendhal ont en commun, c'est le même sentiment de la solitude de l'individu à l'époque contemporaine, alors que la société s'est recomposée, ou plutôt décomposée. Cela se traduit, dans un cas, par les gémissements et les soupirs mélancolique du héros, dans l'autre, par la confrontation énergique du jeune homme avec l'ordre social, comprend-on à la lecture du compte rendu proprement dit du roman de Stendhal.

À la suite de ce développement liminaire, le critique enchaîne :

M. de Stendhal sent que les êtres les mieux organisés sont les plus maltraités par la société qui leur refuse leur place; à ses yeux, il y a autant de perversité et mille fois plus de bassesse dans cet honnête marchand hollandais qui emploie toutes ses facultés pour acquérir sans vol cet or, unique thermomètre auquel tout se mesure, que dans ce brigand qui tue un homme pour la fantaisie de s'emparer de sa maitresse ou de son fusil. Il va nous peindre un de ces hommes au cœur plus grand que leur fortune, qui veulent conquérir le poste auquel ils ont droit, et qui, en duel avec la société, tombent frappés par la foudre. (p. 574-575)

On est ici au cœur de la poétique stendhalienne de l'énergie, du culte héroïque des «caractères» (p. 574), comme Julien et Mathilde dans le roman.

Dans la droite ligne des considérations idéologiques et littéraires si bien exposées dans Le Globe, nous aborderons pour finir la question qui a le plus irrité les critiques contemporains de Stendhal, celle du titre. La plupart d'entre eux se contentent, en faisant montre de plus ou moins d'esprit, qu'ils n'y comprennent rien, et affectent de ne pas chercher une signification à ce qui manifestement à leurs yeux n'en a pas. Pour la plupart, ils s'en tiennent à une formule du genre: «À chacun sa couleur et son style» (p. 617). Deux comptes rendus seulement essaient vraiment de dire quelque chose sur le titre, celui du Journal des Débats, et celui du Temps. L'explication de Janin dans le Journal des Débats est un peu courte, mais non sans intérêt : d'après lui, le roman aurait dû s'appeler Le Jésuite et le Bourgeois ou Les Libéraux et la Congrégation (p. 590), 
et Stendhal a désigné cette opposition par les «couleurs emblématiques» du rouge et du noir. Cette explication aura la fortune que l'on sait, mais elle a le mérite en i 830 de mettre au centre de la lecture du roman la dimension historique et politique. Réflexion plus fine dans le compte rendu du Temps, que l'on a pu attribuer, non sans raison, à Musset, où on lit ces phrases :

Mais il faut, avant de poser la plume, parler de ce style qui, comme le dit l'annonce, est tantôt noir jusqu'au lugubre, tantôt rouge comme du sang. Les caractères ont aussi ces deux nuances, et bien marquées. C'est sans doute là ce que promet le titre, si tant est qu'il promette quoi que ce soit. Du reste, M. de Stendhal est un désenchanteur par excellence; il aime à désoler son monde : il affectionne l'imprévu. (p. 706)

C'est fort bien vu, et pour une fois on a une lecture intelligente, sinon une explication, du titre du roman. L'opposition entre rouge et noir est référée au style et dans cette opposition tranchée c'est le caractère désenchanteur de Stendhal qui s'exprime; désenchantement philosophique et idéologique : est-il nécessaire de le préciser? Le critique du Figaro, sans s'interroger sur le titre, va dans le même sens. Il commence par évoquer «les époques de transition» (p. 585 ) et fait une esquisse polémique de l'après-Juillet, pour conclure sa diatribe par la question : «quelle sympathie devez-vous attendre d'une époque qui ne connaît plus vos modèles, qui a crevé votre toile avec un pavé, sali vos couleurs avec la boue de juillet?» S'agissant d'un roman dont le chromatisme est un élément essentiel, cette remarque est fort bien venue et contribue à lier littérature et politique. Cela débouche sur des considérations de style résumées en deux phrases à la fin de l'article :

[...] tout ce qui est causeries, aperçus, portraits, raillerie, philosophie, impiété, dans le roman de M. de Stendhal, y est traité avec supériorité. Calme sans être mesuré, le style en est jeune, frais, et plein de couleur, trop plein quelquefois, car c'est le vermillon qui domine. (p. 588)

D'un côté, l'héritage du XVIII ${ }^{\mathrm{e}}$ siècle, d'autre part, le vermillon, c'est-à-dire un rouge éclatant. Est-ce un rouge romantique cependant, est-ce le rouge de Delacroix? Ou, pour autrement dire, quel est le style de ce rouge? La difficulté pour les critiques est justement que ce rouge du Ronge et le Noir ne participe pas du chromatisme romantique et qu'il est d'une autre nature. Dans l'usage qu'en fait Stendhal, c'est une manière différente.

Nous soutiendrons en conclusion que dans la question du style telle qu'elle est posée par le plus grand nombre des critiques de i 830, c'est à la fois le rapport de Stendhal au roman et le rapport du roman lui-même à la réalité qui est envisagé. Ce rapport le plus souvent n'est pas appréhendé en termes littéraires, même si, en creux, ou en arrière-plan, s'entr'aperçoit le débat sur 
le romantisme. Une chose est certaine : lorsque les critiques parlent du style du Rouge et le Noir, ils réagissent idéologiquement à la représentation de la réalité que donne le roman. Le critique de la Revue de Paris écrit, par exemple, cette phrase pleine de sens : «si la lecture de pareilles fictions vous laisse le cœur serré et malade d'un horrible désenchantement, que serait-ce donc de la réalité, si elle existait?» (p. 602). Accuser Stendhal, comme c'est souvent fait dans les comptes rendus, d'être un désenchanteur, un briseur d'illusions, en adoptant notamment un style qui n'est pas celui des romans sentimentaux, mais qui témoigne d'une exigence d'analyse et de rigueur, cela revient à dénoncer chez lui une attitude qui détone, avec un seul n. Le mauvais ton qu'on lui reproche implicitement consiste à ne pas jouer le jeu, littéraire ou politique, peu importe; ce qu'on lui reproche, c'est de manquer de manières. Mérimée le dira très crûment à son ami; ce qu'on ne lui pardonnera pas, «c'est d'avoir exposé à nu et au grand jour certaines plaies du cœur humain trop salopes pour être vues ${ }^{3} »$. Avouons que si la formulation n'est pas très beyliste, elle est très stendhalienne, bien accordée en tout cas à un roman qui prétend dire «la vérité, l'âpre vérité» du XIX ${ }^{e}$ siècle.

3. Mérimée, lettre de décembre i830, dans Stendhal, Correspondance, Champion, I997I 999, t. III, p. 526. 
